\title{
A Proactive Approach for Detecting Ransomware based on Hidden Markov Model (HMM)
}

\author{
Mohammed A. Saleh \\ Computer Science Department, College of Sciences and Arts in Ar Rass \\ Qassim University, Kingdom of Saudi Arabia (KSA)
}

\begin{abstract}
A ransomware is the most hazardous kind of computer malware that causes a huge devastation to the computer systems, so that detecting it is highly required at the moment. Truthfully, several prior researchers addressed Markov Model and its variants, like Hidden Markov Model, to detect a malware, but none of them addressed the detection of ransomware through Assembly language instructions. In this paper, a new proactive approach for detecting ransomware based on Hidden Markov Model (HMM) is proposed in order to detect and classify ransomware. In addition, new datasets that comprises of various benign and ransomware are generated and collected. The proposed approach utilized Hidden Markov Model (HMM) for analyzing the generated and collected datasets from benign and ransomware samples, and it detected and classified all samples correctly with $73 \%$ accurate testing samples emissions sequence.
\end{abstract}

\section{Introduction}

In recent times, ransomware attacks form the extremely active and widely spread waves of malware attacks [1-2]. Ransomware is a kind of malware, which is maliciously attacks the victim and encrypts his digital objects, like files and folders, and enforces and extorts the victim to pay a ransom, such as a money or bitcoins, in order to decrypt his digital objects, and therefore; the victim gets his files and folders back [3-5]. Although a ransomware is one type of the malware, it differs than other types of malware in many features, such as the huge amount of digital objects, files and folders, processing for encrypting them and wiping the original copies completely, and eventually, it orders a ransom to the victim. Indeed, a ransomware constitutes an extreme havoc to computers systems, hence; it should be detected and classified preciously in order to avoid its negative effects [6-7]. In reality, a ransomware infects victims through a bug, like zero-days vulnerabilities, or social engineering tactics [8-10].

In this paper, a new proactive approach for detecting ransomware based on Hidden Markov Model (HMM) is proposed to tackle ransomware in order to detect and classify them properly [11]. The proposed approach is a proactive, since it is crucial to classify the sample before the execution under a computer system. In case the approach detects and classifies it as a ransomware, it blocks it immediacy, or otherwise allows it to execute [12]. Besides that, the approach utilizes reinforcement of machine learning, namely Hidden Markov Model (HMM), to train and test the generated and collected datasets [13-15].

This research paper is organized as follows: section 1 presents introduction. Sections 2 discusses the literature review, which involves definitions and theory of Hidden Markov Model (HMM), and previous related works. Section 3 demonstrates generating and collecting training and testing datasets. Section 4 explains a proactive approach for detecting ransomware based on Hidden Markov Model (HMM). Section 5 presents the empirical results and discusses their analyses accordingly. Finally, section 6 presents the conclusion and future work.

\section{Literature review}

\subsection{Hidden Markov Model (HMM)}

A Markov Model is a stochastic process that describes the probabilities of sequences of random variables and states [11][14]. It makes very strong assumption that when predicting the future, it only depends on the present, and the past does not matter at all, as labelled in the next equation 1 .

$$
P\left(S_{i k} \mid S_{i 1}, S_{i 2}, \ldots, S_{i k-1}\right)=P\left(S_{i k} \mid S_{i k-1}\right)
$$

where $\{\mathrm{S} 1, \mathrm{~S} 2, \ldots, \mathrm{SN}\}$ are a set of states. In many cases, the variables and states (classes) that we are interested in are not observable directly, which are hidden, and therefore; Hidden Markov Model is used, since it used to describe the both: observable and hidden variables and states (classes). According to [16-17] the Hidden Markov models is characterized by three essential problems:

- $\quad$ Problem 1 (Evaluation/Likelihood): aims to determine the evaluation (likelihood) based on the following equations: equation 2 , equation 3 , and equation 4.

$$
\mathrm{P}(\mathrm{O} \mid \lambda)=\sum_{\mathrm{i}=1}^{\mathrm{N}} \alpha_{\mathrm{T}}(\mathrm{i})
$$




$$
\begin{aligned}
& \alpha_{\mathrm{t}}(\mathrm{j})=\sum_{\mathrm{i}=1}^{\mathrm{N}} \alpha_{\mathrm{t}-1}(\mathrm{i}) a_{i j} b_{j}\left(o_{t}\right) \\
& \alpha_{1}(\mathrm{j})=\pi_{j} b_{j}\left(o_{1}\right)
\end{aligned}
$$

- $\quad$ Problem 2 (Decoding): seeks to discover the best hidden state sequence, and it uses the succeeding equations: equation 5 , equation 6 , and equation 7.

$$
\begin{aligned}
& \text { Best Score } \mathrm{P} *=\max _{i=1} N \mathrm{v}_{\mathrm{T}}(\mathrm{i}) \\
& \mathrm{v}_{\mathrm{t}}(\mathrm{j})=\max _{i=1} N \mathrm{v}_{\mathrm{t}-1}(\mathrm{i}) a_{i j} b_{j}\left(o_{t}\right) \\
& \mathrm{v}_{1}(\mathrm{j})=\pi_{j} b_{j}\left(o_{1}\right)
\end{aligned}
$$

- $\quad$ Problem 3 (Learning): trains HMM parameters by calculating the following equations: equation 8 , equation 9 , and equation 10 .

$$
\begin{array}{r}
\mathrm{P}(0 \mid \lambda)=\sum_{\mathrm{i}=1}^{\mathrm{N}} \pi_{\mathrm{j}} b_{\mathrm{j}}\left(o_{1}\right) \beta_{1}(j) \\
\beta_{\mathrm{t}}(j)=\sum_{\mathrm{i}=1}^{\mathrm{N}} a_{i j} b_{j}\left(o_{t+1}\right) \beta_{\mathrm{t}+1}(j) \\
\beta_{\mathrm{T}}(i)=1
\end{array}
$$

where: $\lambda$ : HMM Model, O: Observation Sequence, $\pi \mathrm{i}$ : Initial Probability Distribution, $\alpha \mathrm{t}(\mathrm{j})$ : Forward Path Probability, at-1(j): Previous Forward Path Probability, aij: Transition Probability Matrix, bj(ot): Observation Likelihoods, vt-1: Previous Viterbi Path Probability, $\beta T(j)$ : Backward Probability.

\subsection{Previous related works}

A research done by [13] proposed a classifier based on Hidden Markov Model (HMM) in order to identify the family that a virus belongs to. The research covered Viruses, but not ransomware. Another research conducted by [18] examined Hidden Markov Model (HMM) for four different compilers, hand-written assembly code, three virus construction kits, and a metamorphic virus to state similarities and dissimilarities in the hidden states of the HMM. As well, it developed the dueling HMM Strategy for the creation of improved virus detection tools based on HMMs. The paper covered three virus construction kits and a metamorphic virus, but did cover ransomware. A different research accomplished by [19] applied Hidden Markov Model (HMM) to differentiate between a cyber-security attack and no attack. It breaks the data into three clusters using Fuzzy K mean (FKM), after it that labels a small data manually (Analyst Intuition), and lastly it uses HMM state-based approach. The study tackled merely network attacks. A research prepared by [12] created a HMM-based models for each malware family based on its sequence of system calls. The research treated almost all types of malware, except ransomwares. Another relevant research [20] compared API call sequences and opcode sequences using the HMM learning model in order to detect malware. The paper did not study any ransomware sample. A related research work [21] proposed a novel malware classification scheme that is based on Hidden Markov Models (HMMs) and discriminative classifiers. The proposed scheme takes the sequences of system calls that are generated by malware during execution as observation sequences to train the HMMs. The scheme was not examined and tested towards any ransomware sample. Two similar researches [14][22] introduced a new approach based on machine learning methods with n-gram model for detection malwares. It used Markov blanket method as feature selection technique, reduced size of features. The introduced new approach was not examined and tested against any ransomware sample. A research established by [23] presented a classification technique based on Hidden Markov Model (HMM) in order to classify computer viruses. It tested the presented technique towards used various virus construction tools, and none of them generate ransomware.

\section{Generating and collecting training and testing datasets}

In this paper, datasets are generated and collected from a combination of benign and ransomware samples; 23 benign samples and 22 ransomware samples. The research collected 23 benign samples randomly from a fresh 32-bit and 64-bits Windows operating systems. In the meanwhile, it collected 22 ransomware samples from online malware repositories like [24] and [25]. Subsequently, the whole samples, which involves benign and ransomware samples, are investigated and analyzed in terms of Assembly language instructions in order to explore the sharable Assembly language instructions among the entire samples. The most common sharable Assembly language instructions among the complete samples as discovered by the research are 59 instructions, as follows: mov lea xchg lods pop push call ret leave hlt int add sub div mul inc dec or and xor shr shl test cmp jo jno js jns je jz jne jnz jb jnae jc jnb jae jnc jbe jna ja jnbe jl jnge jge jnl jle jng jg jnle jp jpe jnp jpo jcxz jecxz jmp loop nop.

Afterward, these discovered 59 Assembly language instructions are grouped and clustered according to the type of instruction operation, which includes 5 different operation types, namely Data Processing Instructions (Opcodes), Process Instructions (Opcodes), Arithmetic Instructions (Opcodes), Logic Instructions (Opcodes), and Control Flow Instructions (Opcodes). The 59 Assembly language instructions are grouped and 
clustered based on its equivalent operation type as the following: 6 Data Processing Instructions (Opcodes) involve mov lea xchg lods pop push, 5 Process Instructions (Opcodes) include call ret leave hlt int, 5 Arithmetic Instructions (Opcodes) contain add sub div mul inc dec, 6 Logic Instructions (Opcodes) encompass or and xor shr shl test, and 36 Control Flow Instructions (Opcodes) comprise cmp jo jno js jns je jz jne jnz jb jnae jc jnb jae jnc jbe jna ja jnbe jl jnge jge jnl jle jng jg jnle jp jpe jnp jpo jcxz jecxz jmp loop nop.

The benign and ransomware samples, executable Windows software, are converted to Assembly language instructions (Opcodes), and then occurrences of the Opcodes are counted according to the operation types that are listed above. Table 1 and Table 2, as shown in see Appendix 1 and Appendix 2 , present the overall generated and collected datasets from benign and ransomware samples, respectively.

\section{A proactive approach for detecting ransomware based on Hidden Markov Model (HMM)}

Practically, the proposed proactive approach trains Hidden Markov Model (HMM) throughout Problem 3, Problem 1, and Problem 2 of the HMM model using the benign and ransomware training datasets, which are presented in Table 3 and Table 4, in order to identify benign profile and ransomware profile. The benefit of these profiles is that they will be used as benchmarks for classifying testing dataset later on. The overall datasets, which are shown in Table 1 and Table 2, are fragmented randomly into training dataset and testing dataset based on $80 \%$ for training dataset and $20 \%$ for testing dataset. The following Table 3 displays the training dataset from benign samples, while Table 4 presents the training dataset from ransomware samples. Table 5 shows the testing dataset from benign and ransomware samples. The training and testing processes of Hidden Markov Model (HMM) are demonstrated in Figure 1 and Figure 2, correspondingly.

Table 3. Training dataset from benign samples

\begin{tabular}{|c|c|c|c|c|c|c|c|}
\hline No & $\begin{array}{c}\text { Benign } \\
\text { Software/Program }\end{array}$ & $\begin{array}{c}\text { Data } \\
\text { Processing } \\
\text { Opcodes }\end{array}$ & $\begin{array}{l}\text { Process } \\
\text { Opcodes }\end{array}$ & $\begin{array}{l}\text { Arithmetic } \\
\text { Opcodes }\end{array}$ & $\begin{array}{c}\text { Logic } \\
\text { Opcodes }\end{array}$ & $\begin{array}{c}\text { Control } \\
\text { Flow } \\
\text { Opcodes }\end{array}$ & Total \\
\hline 1 & ComputerDefaults.exe & 2217 & 906 & 3894 & 784 & 1801 & 9602 \\
\hline 2 & DisplaySwitch.exe & 27806 & 9648 & 59453 & 8947 & 24779 & 130633 \\
\hline 3 & Magnify.exe & 59064 & 15748 & 42508 & 22518 & 31588 & 171426 \\
\hline 4 & Narrator.exe & 72352 & 4144 & 202347 & 47781 & 69287 & 395911 \\
\hline 5 & calc.exe & 16631 & 3391 & 7405 & 5010 & 4079 & 36516 \\
\hline 6 & clipbrd.exe & 11993 & 3670 & 8097 & 4608 & 6315 & 34683 \\
\hline 7 & cmd.exe & 20227 & 4498 & 13926 & 6514 & 17038 & 62203 \\
\hline 8 & dvdplay.exe & 428 & 311 & 1028 & 291 & 506 & 2564 \\
\hline 9 & freecell.exe & 5632 & 1195 & 6428 & 2645 & 2750 & 18650 \\
\hline 10 & klist.exe & 3034 & 1148 & 2264 & 1150 & 2059 & 9655 \\
\hline 11 & label.exe & 1250 & 468 & 1050 & 435 & 806 & 4009 \\
\hline 12 & mstsc.exe & 79785 & 29979 & 85167 & 29352 & 46704 & 270987 \\
\hline 13 & notepad.exe & 15627 & 3503 & 10828 & 6927 & 11635 & 48520 \\
\hline 14 & ntprint.exe & 4208 & 1060 & 3692 & 2001 & 3030 & 13991 \\
\hline 15 & osk.exe & 41958 & 17077 & 77010 & 12778 & 24615 & 173438 \\
\hline 16 & syskey.exe & 2028 & 1021 & 2316 & 1101 & 1480 & 7946 \\
\hline 17 & taskmgr.exe & 15092 & 4456 & 14411 & 5737 & 7730 & 47426 \\
\hline 18 & winhlp32.exe & 517 & 281 & 911 & 353 & 463 & 2525 \\
\hline 19 & write.exe & 357 & 264 & 821 & 315 & 555 & 2312 \\
\hline \multicolumn{2}{|c|}{ Total } & 380206 & 102768 & 543556 & 159247 & 257220 & 1442997 \\
\hline \multicolumn{2}{|r|}{ Ratio } & 0.2635 & 0.0712 & 0.3767 & 0.1104 & 0.1783 & 1.0000 \\
\hline
\end{tabular}


Table 4. Training dataset from ransomware samples

\begin{tabular}{|c|c|c|c|c|c|c|c|}
\hline No & Ransomware & $\begin{array}{c}\text { Data } \\
\text { Processing } \\
\text { Opcodes }\end{array}$ & $\begin{array}{l}\text { Process } \\
\text { Opcodes }\end{array}$ & $\begin{array}{c}\text { Arithmetic } \\
\text { Opcodes }\end{array}$ & $\begin{array}{c}\text { Logic } \\
\text { Opcodes }\end{array}$ & $\begin{array}{l}\text { Control } \\
\text { Flow } \\
\text { Opcodes }\end{array}$ & Total \\
\hline 1 & cerber.exe & 27087 & 5423 & 24903 & 16281 & 155477 & 229171 \\
\hline 2 & cryptowall.exe & 21993 & 1505 & 50955 & 13102 & 16373 & 103928 \\
\hline 3 & locky.exe & 15425 & 5158 & 16462 & 10435 & 12260 & 59740 \\
\hline 4 & mamba.exe & 233132 & 53856 & 232065 & 154467 & 133771 & 807291 \\
\hline 5 & matsnu.exe & 9755 & 2653 & 13174 & 4838 & 5824 & 36244 \\
\hline 6 & petrwrap 1.exe & 42976 & 8020 & 21662 & 19668 & 22007 & 114333 \\
\hline 7 & petrwrap 2.exe & 60185 & 8278 & 103806 & 34585 & 41059 & 247913 \\
\hline 8 & petrwrap.exe & 42976 & 8020 & 21662 & 19668 & 22007 & 114333 \\
\hline 9 & petya 1.exe & 96530 & 23008 & 54206 & 35338 & 45482 & 254564 \\
\hline 10 & petya.exe & 96530 & 23008 & 54206 & 35338 & 42333 & 251415 \\
\hline 11 & radaman_UPX.ViR.exe & 0 & 0 & 0 & 1 & 0 & 1 \\
\hline 12 & satana.exe & 8538 & 443 & 9593 & 3906 & 4516 & 26996 \\
\hline 13 & teslacrypt 1.exe & 26309 & 5063 & 21494 & 10583 & 13831 & 77280 \\
\hline 14 & teslacrypt 2.exe & 40067 & 9083 & 15219 & 14111 & 18316 & 96796 \\
\hline 15 & vipasana 1.exe & 55799 & 10582 & 25264 & 26035 & 22068 & 139748 \\
\hline 16 & vipasana 3.exe & 51241 & 9714 & 22437 & 22000 & 19819 & 125211 \\
\hline 17 & wannacry.exe & 393909 & 68355 & 205262 & 179467 & 211577 & 1058570 \\
\hline \multicolumn{2}{|c|}{ Total } & 1516765 & 292154 & 1059744 & 734662 & 946823 & 4550148 \\
\hline & Ratio & 0.3333 & 0.0642 & 0.2329 & 0.1615 & 0.2081 & 1.0000 \\
\hline
\end{tabular}

Table 5. Testing dataset from benign and ransomware samples

\begin{tabular}{|c|c|c|c|c|c|c|c|}
\hline No & $\begin{array}{c}\text { Benign } \\
\text { Software/Program }\end{array}$ & $\begin{array}{c}\text { Data } \\
\text { Processing } \\
\text { Opcodes }\end{array}$ & $\begin{array}{c}\text { Process } \\
\text { Opcodes }\end{array}$ & $\begin{array}{c}\text { Arithmetic } \\
\text { Opcodes }\end{array}$ & $\begin{array}{c}\text { Logic } \\
\text { Opcodes }\end{array}$ & $\begin{array}{c}\text { Control Flow } \\
\text { Opcodes }\end{array}$ & Total \\
\hline 1 & Defrag.exe & 16424 & 4116 & 10009 & 7056 & 9654 & 47259 \\
\hline 2 & Ratio & 0.3475 & 0.0871 & 0.2118 & 0.1493 & 0.2043 & 1.0000 \\
\hline & petya 2.exe & 32682 & 6592 & 17918 & 10897 & 13303 & 81392 \\
\hline 3 & Ratio & 0.4015 & 0.0810 & 0.2201 & 0.1339 & 0.1634 & 1.0000 \\
\hline & colorcpl.exe & 6861 & 1885 & 5526 & 3246 & 4324 & 21842 \\
\hline 4 & Ratio & 0.3141 & 0.0863 & 0.2530 & 0.1486 & 0.1980 & 1.0000 \\
\hline & teslacrypt 3.exe & 26848 & 5073 & 21489 & 10788 & 14089 & 78287 \\
\hline 5 & Ratio & 0.3429 & 0.0648 & 0.2745 & 0.1378 & 0.1800 & 1.0000 \\
\hline & wannacry+.exe & 294313 & 49985 & 167374 & 134839 & 160103 & 806614 \\
\hline 6 & Ratio & 0.3649 & 0.0620 & 0.2075 & 0.1672 & 0.1985 & 1.0000 \\
\hline & resmon.exe & 8543 & 2199 & 5670 & 4151 & 5261 & 25824 \\
\hline 7 & Ratio & 0.3308 & 0.0852 & 0.2196 & 0.1607 & 0.2037 & 1.0000 \\
\hline & mblctr.exe & 65404 & 19910 & 62871 & 26866 & 46543 & 221594 \\
\hline 8 & Ratio & 0.2952 & 0.0898 & 0.2837 & 0.1212 & 0.2100 & 1.0000 \\
\hline & vipasana 2.exe & 55622 & 10551 & 25397 & 26058 & 22199 & 139827 \\
\hline
\end{tabular}




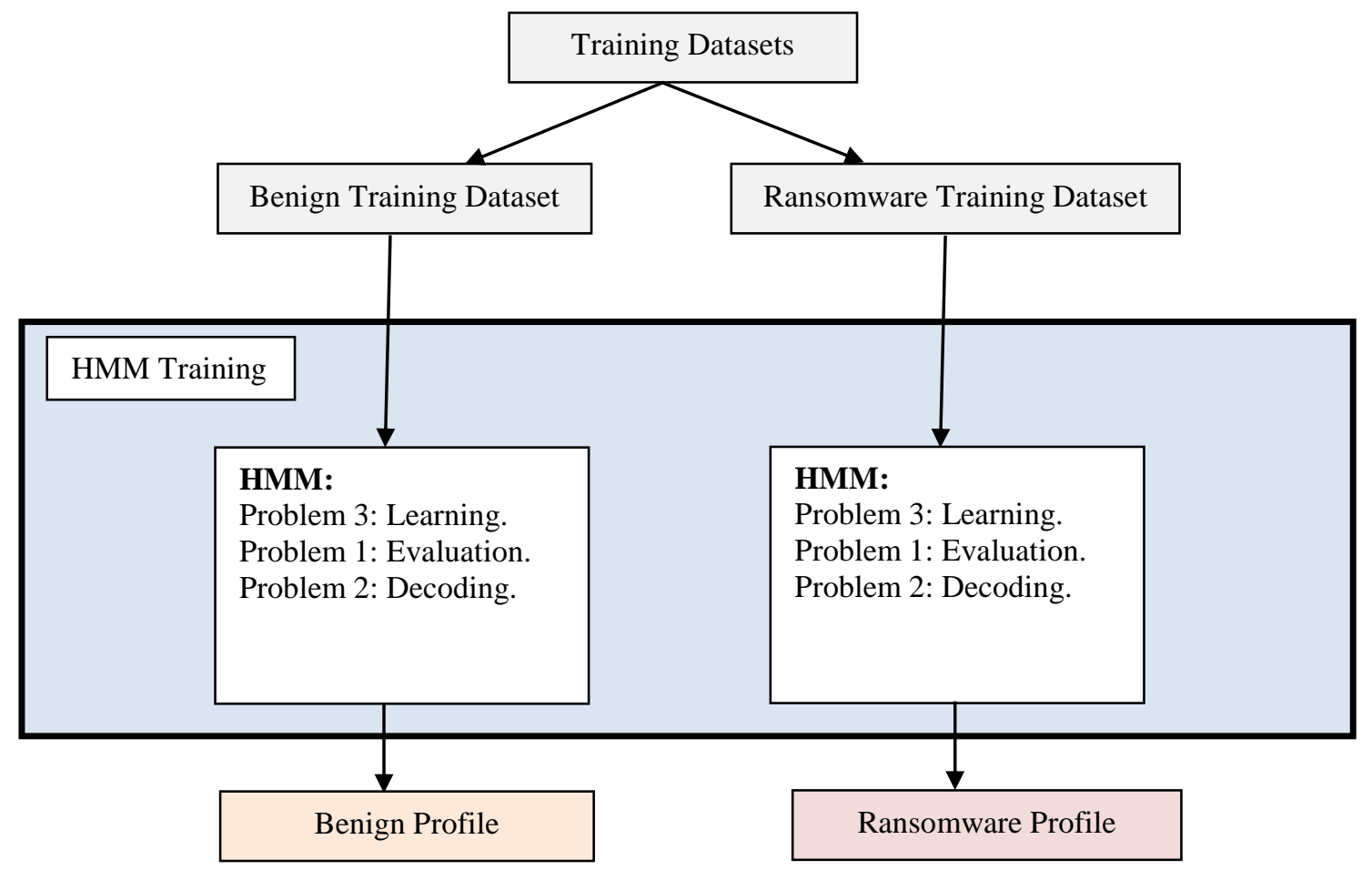

Figure 1. Training processes of Hidden Markov Model (HMM)

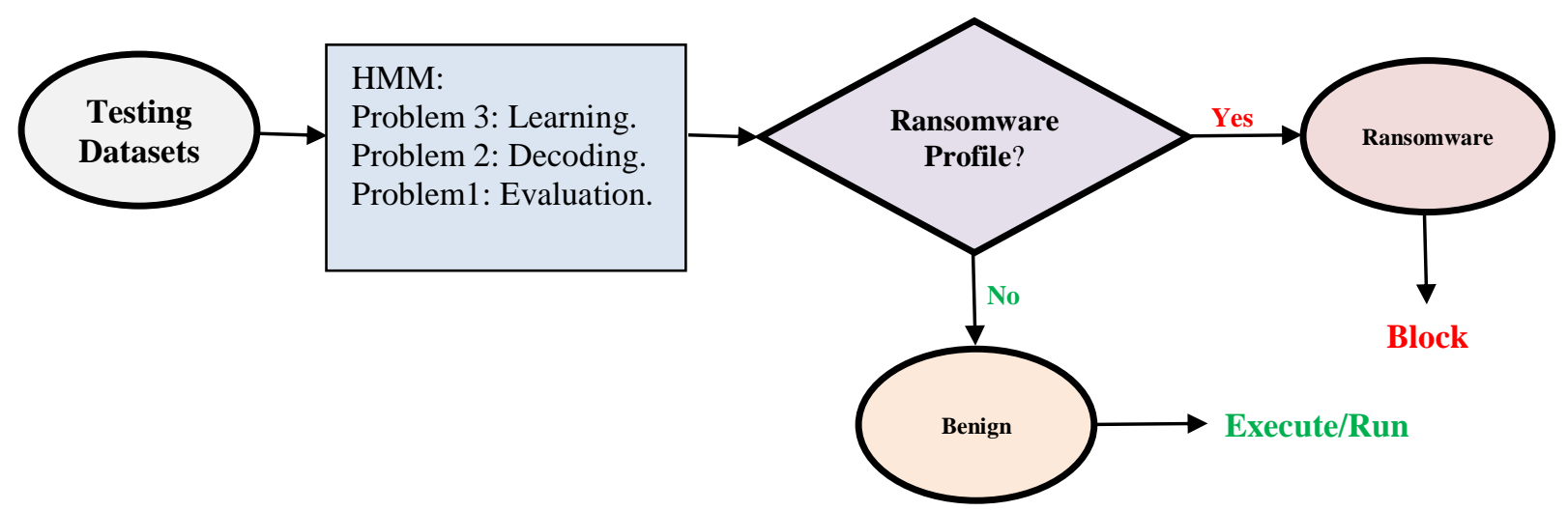

Figure 2. Testing processes of Hidden Markov Model (HMM)

\section{Empirical results and discussions}

The empirical for testing the proposed proactive approach for detecting ransomware based on Hidden Markov Model (HMM) are conducted on MATLAB software [26] for the sake of analysing training and testing datasets, which are generated and collected from benign and ransomware samples. First, the empirical computed Problem 3, which is training HMM model, twice for both training datasets of benign samples, in Table 3, and ransomware samples, in Table 4, as depicted in Figure 1. The results are shown as the following:

Ransomware Transition Matrix

$$
=\left[\begin{array}{ll}
0.9000 & 0.1000 \\
0.0500 & 0.9500
\end{array}\right]
$$

Ransomware Emissions Matrix

$=\left[\begin{array}{ccccc}0 & 0 & 0 & 0 & 0 \\ 0.3333 & 0.0642 & 0.2329 & 0.1615 & 0.2081\end{array}\right]$

Estimated (Trained) Ransomware Transition Matrix $=\left[\begin{array}{ll}0 & 1 \\ 0 & 1\end{array}\right]$ 
Estimated (Trained) Ransomware Emissions Matrix

$=\left[\begin{array}{ccccc}0 & 0 & 0 & 0 & 0 \\ 0.2000 & 0.2000 & 0.2000 & 0.2000 & 0.2000\end{array}\right]$

Ransomware Emissions Sequence

$$
=\left[\begin{array}{lllll}
1 & 3 & 5 & 4 & 2
\end{array}\right]
$$

Benign Transition Matrix $=\left[\begin{array}{ll}0.9000 & 0.1000 \\ 0.0500 & 0.9500\end{array}\right]$

Benign Emissions Matrix

$=\left[\begin{array}{ccccc}0.2635 & 0.0712 & 0.3767 & 0.1104 & 0.1783 \\ 0 & 0 & 0 & 0 & 0\end{array}\right]$

Estimated (Trained) Benign Transition

Matrix $=\left[\begin{array}{ll}1 & 0 \\ 0 & 1\end{array}\right]$

Estimated (Trained) Benign Emissions Matrix

$=\left[\begin{array}{ccccc}0.2000 & 0.2000 & 0.2000 & 0.2000 & 0.2000 \\ 0 & 0 & 0 & 0 & 0\end{array}\right]$

Benign Emissions Sequence $=\left[\begin{array}{lllll}3 & 1 & 5 & 4 & 2\end{array}\right]$

Second, the empirical calculated Problem 1, which is an evaluation and a likelihood, and Problem 2, which is a decoding, of the HMM model twice for both training datasets of benign samples, in Table 3, and ransomware samples, in Table 3 , as illustrated in Figure 1. The aim of these two steps is to construct benign profile and ransomware profile, which they will be used as benchmarks in testing process later on. The results are revealed in the next:
Ransomawre Posterior State Probabilities

$$
=\left[\begin{array}{lllll}
0 & 0 & 0 & 0 & 0 \\
1 & 1 & 1 & 1 & 1
\end{array}\right]
$$

Ransomawre Best State Path (Viterbi)

$$
=\left[\begin{array}{lllll}
2 & 2 & 2 & 2 & 2
\end{array}\right]
$$

Benign Posterior State Probabilities

$$
=\left[\begin{array}{lllll}
1 & 1 & 1 & 1 & 1 \\
0 & 0 & 0 & 0 & 0
\end{array}\right]
$$

Benign Best State Path (Viterbi)

$$
=\left[\begin{array}{lllll}
1 & 1 & 1 & 1 & 1
\end{array}\right]
$$

Finally, the empirical computed Problem 3, Problem 2, and Problem 1 of the HMM model for testing dataset of benign and ransomware samples in Table 5 randomly, as explained in Figure 2. Then, the gained result is compared to the ransomware profile, and in case it matches the profile, the sample is classified as a ransomware, or otherwise is classified as a benign software. The proposed proactive approach for detecting ransomware based on Hidden Markov Model (HMM) detected and classified all ransomware samples in the testing dataset precisely with $85 \%$ accurate ransomware testing samples emissions sequence. As well, it detected and classified all benign samples in the testing dataset exactly with $60 \%$ accurate benign

\begin{tabular}{|c|c|c|c|c|c|}
\hline No & Program & $\begin{array}{l}\text { Posterior State } \\
\text { Probabilities }\end{array}$ & $\begin{array}{l}\text { Emissions } \\
\text { Sequence }\end{array}$ & $\begin{array}{c}\text { Best State Path } \\
\text { (Viterbi) }\end{array}$ & Classification \\
\hline 1 & Defrag.exe & {$\left[\begin{array}{lllll}1 & 1 & 1 & 1 & 1\end{array}\right]$} & {$\left[\begin{array}{lllll}1 & 3 & 5 & 4 & 2\end{array}\right]$} & {$\left[\begin{array}{lllll}1 & 1 & 1 & 1 & 1\end{array}\right]$} & Benign \\
\hline 2 & petya 2.exe & {$\left[\begin{array}{lllll}2 & 2 & 2 & 2 & 2\end{array}\right]$} & {$\left[\begin{array}{lllll}1 & 3 & 5 & 4 & 2\end{array}\right]$} & {$\left[\begin{array}{lllll}2 & 2 & 2 & 2 & 2\end{array}\right]$} & Ransomware \\
\hline 3 & colorcpl.exe & {$\left[\begin{array}{lllll}1 & 1 & 1 & 1 & 1\end{array}\right]$} & {$\left[\begin{array}{lllll}1 & 3 & 5 & 4 & 2\end{array}\right]$} & {$\left[\begin{array}{lllll}1 & 1 & 1 & 1 & 1\end{array}\right]$} & Benign \\
\hline 4 & teslacrypt 3.exe & {$\left[\begin{array}{lllll}2 & 2 & 2 & 2 & 2\end{array}\right]$} & {$\left[\begin{array}{lllll}1 & 3 & 5 & 4 & 2\end{array}\right]$} & {$\left[\begin{array}{lllll}2 & 2 & 2 & 2 & 2\end{array}\right]$} & Ransomware \\
\hline 5 & wannacry+.exe & {$\left[\begin{array}{lllll}2 & 2 & 2 & 2 & 2\end{array}\right]$} & {$\left[\begin{array}{lllll}1 & 3 & 5 & 4 & 2\end{array}\right]$} & {$\left[\begin{array}{lllll}2 & 2 & 2 & 2 & 2\end{array}\right]$} & Ransomware \\
\hline 6 & resmon.exe & {$\left[\begin{array}{lllll}1 & 1 & 1 & 1 & 1\end{array}\right]$} & {$\left[\begin{array}{lllll}1 & 3 & 5 & 4 & 2\end{array}\right]$} & {$\left[\begin{array}{lllll}1 & 1 & 1 & 1 & 1\end{array}\right]$} & Benign \\
\hline 7 & mblctr.exe & {$\left[\begin{array}{lllll}1 & 1 & 1 & 1 & 1\end{array}\right]$} & {$\left[\begin{array}{lllll}1 & 3 & 5 & 4 & 2\end{array}\right]$} & {$\left[\begin{array}{lllll}1 & 1 & 1 & 1 & 1\end{array}\right]$} & Benign \\
\hline 8 & vipasana 2.exe & {$\left[\begin{array}{lllll}2 & 2 & 2 & 2 & 2\end{array}\right]$} & {$\left[\begin{array}{lllll}1 & 4 & 3 & 5 & 2\end{array}\right]$} & {$\left[\begin{array}{lllll}2 & 2 & 2 & 2 & 2\end{array}\right]$} & Ransomware \\
\hline
\end{tabular}
testing samples emissions sequence, with overall $73 \%$ accurate testing samples emissions sequence, as shown in Table 6 and Table 7.

Table 6. The results of Hidden Markov Model (HMM) on testing dataset samples

Table 7. The percentage of detection and classification for testing dataset samples

\begin{tabular}{|c|c|c|c|c|}
\hline No & Classification & $\begin{array}{c}\text { Detection and Classification } \\
\text { Percentage }\end{array}$ & \multicolumn{2}{|c|}{ Emissions Sequence Percentage } \\
\hline 1 & Benign & $100 \%$ & $60 \%$ & $73 \%$ \\
\hline 2 & Ransomware & $100 \%$ & $85 \%$ & \\
\hline
\end{tabular}




\section{Conclusion}

This research paper proposed a new proactive approach for detecting ransomware based on Hidden Markov Model (HMM). The approach is a proactive, since it firstly analyzes the samples, and in case it classifies it as a ransomware, it will detect and eliminate it before the execution. It generated and collected training and testing datasets from various benign and ransomware samples. In the meantime, it investigated and analyzed the samples in terms of Assembly language instructions, or Opcodes, in order to explore the sharable Assembly language instructions among the entire samples. The most common sharable Assembly language instructions among the complete samples as discovered by the research were 59 instructions, which were grouped and clustered according to the type of instruction operation, which includes 5 different operation types, namely Data Processing Instructions (Opcodes), Process Instructions (Opcodes), Arithmetic Instructions (Opcodes), Logic Instructions (Opcodes), and Control Flow Instructions (Opcodes). The approach identified and constructed benign profile and ransomware profile, which will be used as benchmarks for classifying testing dataset later on. Empirically, the proposed approach detected and classified all ransomware samples in the testing dataset precisely with $85 \%$ accurate ransomware testing samples emissions sequence. As well, it detected and classified all benign samples in the testing dataset exactly with $60 \%$ accurate benign testing samples emissions sequence, with overall $73 \%$ accurate testing samples emissions sequence. Hopefully the future works will expand this research to involve more ransomware samples.

\section{References}

[1] P. Paganini, "Thousands of servers infected with the Lilocked Ransomware," securityaffairs.co, 07-Sep-2019.

[2] C. Cimpanu, "Ransomware gang wanted $\$ 5.3$ million from US city, but they only offered \$400,000," www.zdnet.com, 2019. [Online]. Available:

https://www.zdnet.com/article/ransomware-gangwanted-5-3-million-from-us-city-but-they-onlyoffered-400000/. [Accessed: 13-Sep-2019].

[3] C. Cimpanu, "Thousands of servers infected with new Lilocked (Lilu) ransomware," www.zdnet.com, 2019. [Online]. Available: https://www.zdnet.com/article/thousands-ofservers-infected-with-new-lilocked-liluransomware/. [Accessed: 13-Sep-2019].

[4] I. Ilascu, "Fake PayPal Site Spreads Nemty Ransomware," www.bleepingcomputer.com, 2019. [Online]. Available: https://www.bleepingcomputer.com/news/security /fake-paypal-site-spreads-nemty-ransomware/. [Accessed: 13-Sep-2019].
[5] S. Morgan, "Global Ransomware Damage Costs Predicted To Exceed \$5 Billion In 2017," May2017.

[6] K. P. Subedi, D. R. Budhathoki, and D. Dasgupta, "Forensic Analysis of Ransomware Families Using Static and Dynamic Analysis," 2018 IEEE Secur. Priv. Work., pp. 180-185, 2018.

[7] E. Kirda, "UNVEIL: A large-scale, automated approach to detecting ransomware (keynote)," 2017 IEEE 24th Int. Conf. Softw. Anal. Evol. Reengineering, pp. 1-1, 2017.

[8] S. Poudyal, K. P. Subedi, and D. Dasgupta, "A Framework for Analyzing Ransomware using Machine Learning," in 2018 IEEE Symposium Series on Computational Intelligence (SSCI), 2018, pp. 1692-1699.

[9] D. Y. Kim, G. Y. Choi, and J. H. Lee, "White listbased ransomware real-time detection and prevention for user device protection," 2018 IEEE Int. Conf. Consum. Electron. ICCE 2018, vol. 2018-Janua, pp. 1-5, 2018.

[10] M. M. Ahmadian and H. R. Shahriari, "2entFOX: A framework for high survivable ransomwares detection," 13th Int. ISC Conf. Inf. Secur. Cryptology, Isc. 2016, pp. 79-84, 2016.

[11] S. K. Sasidharan and C. Thomas, "A Survey on Metamorphic Malware Detection based on Hidden Markov Model," 2018 Int. Conf. Adv. Comput. Commun. Informatics, ICACCI 2018, pp. 357-362, 2018.

[12] R. Pranamulia, Y. Asnar, and R. S. Perdana, "Profile Hidden Markov Model for Malware Classification - Usage of System call Sequence for Malware Classification," 2017.

[13] S. P. Thunga and R. K. Neelisetti, "Identifying metamorphic virus using n-grams and Hidden Markov Model," 2015 Int. Conf. Adv. Comput. Commun. Informatics, ICACCI 2015, pp. 20162022, 2015.

[14] B. Pechaz, M. V. Jahan, and M. Jalali, "Malware detection using hidden markov model based on markov blanket feature selection method," 2nd Int. Congr. Technol. Commun. Knowledge, ICTCK 2015, no. Ictck, pp. 558-563, 2016.

[15] K. Xin, G. Li, Z. Qin, and Q. Zhang, "Malware detection in smartphone using Hidden Markov Model," Proc. - 2012 4th Int. Conf. Multimed. Secur. MINES 2012, pp. 857-860, 2012.

[16] D. Jurafsky and J. H. Martin, An Introduction to Natural Language Processing, Computational Linguistics, and Speech Recognition, Third Edit. 2018.

[17] L. R. Rabiner, "A tutorial on hidden Markov models and selected applications in speech recognition.," in Proceedings of the IEEE, 77(2), 1989, pp. 257-286.

[18] T. H. Austin, E. Filiol, S. Josse, and M. Stamp, "Exploring hidden Markov models for virus analysis: A semantic approach," Proc. Annu. Hawaii Int. Conf. Syst. Sci., pp. 5039-5048, 2013.

[19] T. T. Teoh, Y. Y. Nguwi, Y. Elovici, N. M. Cheung, and W. L. Ng, "Analyst intuition based Hidden Markov Model on high speed, temporal cyber security big data," ICNC-FSKD 2017 - 13th Int. Conf. Nat. Comput. Fuzzy Syst. Knowl. Discov., pp. 2080-2083, 2018. 
[20] S. Alqurashi and O. Batarfi, "A comparison between API call sequences and opcode sequences as reflectors of malware behavior," 2017 12th Int. Conf. Internet Technol. Secur. Trans. ICITST 2017, pp. 105-110, 2018.

[21] M. Imran, M. T. Afzal, and M. A. Qadir, "Similarity-Based Malware Classification Using Hidden Markov Model," Proc. - 4th Int. Conf. Cyber Secur. Cyber Warf. Digit. Forensics, CyberSec 2015, pp. 129-134, 2016.

[22] H. Divandari, B. Pechaz, and M. V. Jahan, "Malware detection using Markov Blanket based on opcode sequences," 2nd Int. Congr. Technol. Commun. Knowledge, ICTCK 2015, no. Ictck, pp. 564-569, 2016.

[23] F. Rezaei, M. Hamedi-Hamzehkolaie, S. Rezaei, and A. Payandeh, "Metamorphic viruses detection by hidden Markov models," 7'th Int. Symp. Telecommun., pp. 821-826, 2015.

[24] Y. Nativ, "theZoo project." [Online]. Available https://github.com/ytisf/theZoo. [Accessed: 15Aug-2019].

[25] CrowdStrike, "reverse." [Online]. Available: https://www.reverse.it/. [Accessed: 15-Aug2019].

[26] MathWorks, “MATLAB.” MathWorks, 2019. 


\section{Appendix 1}

Table 1. The overall generated and collected dataset from benign samples

\begin{tabular}{|c|c|c|c|c|c|c|c|}
\hline No & $\begin{array}{c}\text { Benign } \\
\text { Software/Program }\end{array}$ & $\begin{array}{c}\text { Data } \\
\text { Processing } \\
\text { Opcodes }\end{array}$ & $\begin{array}{l}\text { Process } \\
\text { Opcodes }\end{array}$ & $\begin{array}{c}\text { Arithmetic } \\
\text { Opcodes }\end{array}$ & $\begin{array}{c}\text { Logic } \\
\text { Opcodes }\end{array}$ & $\begin{array}{c}\text { Control } \\
\text { Flow } \\
\text { Opcodes }\end{array}$ & Total \\
\hline 1 & ComputerDefaults.exe & 2217 & 906 & 3894 & 784 & 1801 & 9602 \\
\hline 2 & Defrag.exe & 16424 & 4116 & 10009 & 7056 & 9654 & 47259 \\
\hline 3 & DisplaySwitch.exe & 27806 & 9648 & 59453 & 8947 & 24779 & 130633 \\
\hline 4 & Magnify.exe & 59064 & 15748 & 42508 & 22518 & 31588 & 171426 \\
\hline 5 & Narrator.exe & 72352 & 4144 & 202347 & 47781 & 69287 & 395911 \\
\hline 6 & calc.exe & 16631 & 3391 & 7405 & 5010 & 4079 & 36516 \\
\hline 7 & clipbrd.exe & 11993 & 3670 & 8097 & 4608 & 6315 & 34683 \\
\hline 8 & cmd.exe & 20227 & 4498 & 13926 & 6514 & 17038 & 62203 \\
\hline 9 & colorcpl.exe & 6861 & 1885 & 5526 & 3246 & 4324 & 21842 \\
\hline 10 & dvdplay.exe & 428 & 311 & 1028 & 291 & 506 & 2564 \\
\hline 11 & freecell.exe & 5632 & 1195 & 6428 & 2645 & 2750 & 18650 \\
\hline 12 & klist.exe & 3034 & 1148 & 2264 & 1150 & 2059 & 9655 \\
\hline 13 & label.exe & 1250 & 468 & 1050 & 435 & 806 & 4009 \\
\hline 14 & mblctr.exe & 65404 & 19910 & 62871 & 26866 & 46543 & 221594 \\
\hline 15 & mstsc.exe & 79785 & 29979 & 85167 & 29352 & 46704 & 270987 \\
\hline 16 & notepad.exe & 15627 & 3503 & 10828 & 6927 & 11635 & 48520 \\
\hline 17 & ntprint.exe & 4208 & 1060 & 3692 & 2001 & 3030 & 13991 \\
\hline 18 & osk.exe & 41958 & 17077 & 77010 & 12778 & 24615 & 173438 \\
\hline 19 & resmon.exe & 8543 & 2199 & 5670 & 4151 & 5261 & 25824 \\
\hline 20 & syskey.exe & 2028 & 1021 & 2316 & 1101 & 1480 & 7946 \\
\hline 21 & taskmgr.exe & 15092 & 4456 & 14411 & 5737 & 7730 & 47426 \\
\hline 22 & winhlp32.exe & 517 & 281 & 911 & 353 & 463 & 2525 \\
\hline 23 & write.exe & 357 & 264 & 821 & 315 & 555 & 2312 \\
\hline & Total & 477438 & 130878 & 627632 & 200566 & 323002 & 1759516 \\
\hline
\end{tabular}




\section{Appendix 2}

Table 2. The overall generated and collected dataset from ransomware samples

\begin{tabular}{|c|c|c|c|c|c|c|c|}
\hline No & Ransomware & $\begin{array}{c}\text { Data } \\
\text { Processing } \\
\text { Opcodes }\end{array}$ & $\begin{array}{l}\text { Process } \\
\text { Opcodes }\end{array}$ & $\begin{array}{c}\text { Arithmetic } \\
\text { Opcodes }\end{array}$ & $\begin{array}{c}\text { Logic } \\
\text { Opcodes }\end{array}$ & $\begin{array}{l}\text { Control } \\
\text { Flow } \\
\text { Opcodes }\end{array}$ & Total \\
\hline 1 & cerber.exe & 27087 & 5423 & 24903 & 16281 & 155477 & 229171 \\
\hline 2 & cryptowall.exe & 21993 & 1505 & 50955 & 13102 & 16373 & 103928 \\
\hline 3 & locky.exe & 15425 & 5158 & 16462 & 10435 & 12260 & 59740 \\
\hline 4 & mamba.exe & 233132 & 53856 & 232065 & 154467 & 133771 & 807291 \\
\hline 5 & matsnu.exe & 9755 & 2653 & 13174 & 4838 & 5824 & 36244 \\
\hline 6 & petrwrap 1.exe & 42976 & 8020 & 21662 & 19668 & 22007 & 114333 \\
\hline 7 & petrwrap 2.exe & 60185 & 8278 & 103806 & 34585 & 41059 & 247913 \\
\hline 8 & petrwrap.exe & 42976 & 8020 & 21662 & 19668 & 22007 & 114333 \\
\hline 9 & petya 1.exe & 96530 & 23008 & 54206 & 35338 & 45482 & 254564 \\
\hline 10 & petya 2.exe & 32682 & 6592 & 17918 & 10897 & 13303 & 81392 \\
\hline 11 & petya.exe & 96530 & 23008 & 54206 & 35338 & 42333 & 251415 \\
\hline 12 & radaman_UPX.ViR.exe & 0 & 0 & 0 & 1 & 0 & 1 \\
\hline 13 & satana.exe & 8538 & 443 & 9593 & 3906 & 4516 & 26996 \\
\hline 14 & teslacrypt 1.exe & 26309 & 5063 & 21494 & 10583 & 13831 & 77280 \\
\hline 15 & teslacrypt 2.exe & 40067 & 9083 & 15219 & 14111 & 18316 & 96796 \\
\hline 16 & teslacrypt 3.exe & 26848 & 5073 & 21489 & 10788 & 14089 & 78287 \\
\hline 17 & vipasana 1.exe & 55799 & 10582 & 25264 & 26035 & 22068 & 139748 \\
\hline 18 & vipasana 2.exe & 55622 & 10551 & 25397 & 26058 & 22199 & 139827 \\
\hline 19 & vipasana 3.exe & 51241 & 9714 & 22437 & 22000 & 19819 & 125211 \\
\hline 20 & wannacry+.exe & 294313 & 49985 & 167374 & 134839 & 160103 & 806614 \\
\hline 21 & wannacry.exe & 393909 & 68355 & 205262 & 179467 & 211577 & 1058570 \\
\hline 22 & wannacryPlus.exe & 294313 & 49985 & 167374 & 134839 & 160103 & 806614 \\
\hline \multicolumn{2}{|c|}{ Total } & 1926230 & 364355 & 1291922 & 917244 & 1156517 & 5656268 \\
\hline
\end{tabular}

